\title{
Aspectos socioeconômicos do Perímetro Irrigado Várzeas de Sousa (PIVAS) no Semiárido paraibano
}

\author{
Alexson Vieira Pordeus ${ }^{1}\left(\mathbb{D}\right.$, José Deomar de Souza Barros ${ }^{2}{ }^{\circledR}$, Rafael da Silva \\ Moreira $^{2}\left(\right.$, Jânio Trajano de Araújo ${ }^{1}$ (1) \& José Luciano Andrade da Silva ${ }^{2}(1)$
}

(1) Universidade Federal de Campina Grande, Centro de Ciência e Tecnologia Agroalimentar, Programa de Pós-Graduação em Sistemas Agroindustriais, Rua Jario Vieira Feitosa 1770, Bairro dos Pereiros 58840-000, Pombal, Paraíba, Brasil. E-mail: alexson1.pordeus@gmail.com

(2) Universidade Federal de Campina Grande, Centro de Formação de Professores, Unidade Acadêmica de Ciências Exatas e da Natureza, Rua Sérgio Moreira de Figueiredo, Casas Populares 58900-000, Cajazeiras, Paraíba, Brasil. E-mail: deomarbarros@gmail.com

Pordeus A.V., Barros J.D.S., Moreira R.S., Araújo J.T. \& Silva J.L.A. (2019) Aspectos socioeconômicos do Perímetro Irrigado Várzeas de Sousa (PIVAS) no semiárido paraibano. Pesquisa e Ensino em Ciências Exatas e da Natureza, 3(2): 189-198. http://dx.doi.org/10.29215/pecen.v3i2.1275

Editora acadêmica: Antonia Arisdélia F. M. Aguiar Feitosa. Recebido: 14 Junho 2019. Aceito: 06 Novembro 2019. Publicado: 08 Novembro 2019.

Resumo: As regiões semiáridas apresentam elevados índices de pobreza, quando comparadas as regiões sul e sudeste do Brasil. Este fato aponta a necessidade de adoção de políticas públicas que proporcionem a mitigação dos indicadores de vulnerabilidades socioeconômicas encontrados nessa região. Nesse contexto, o estudo desenvolvido objetivou avaliar os aspectos socioeconômicos do Perímetro Irrigado Várzeas de Sousa, sertão da Paraíba. A pesquisa foi realizada no período de julho de 2016 a agosto de 2017, por meio da aplicação de um formulário junto a 45 pequenos irrigantes. Os resultados obtidos indicam que as propriedades pesquisadas apresentaram um alto índice de vulnerabilidade econômica, apontando para a necessidade de políticas públicas com vista à mitigação desses índices; um baixo índice de vulnerabilidade social, o que pode contribuir para a permanência dos pequenos irrigantes nas atividades agropecuárias.

Palavras chave: Regiões semiáridas, vulnerabilidades socioeconômicas, políticas públicas.

Socioeconomic aspects of the de Sousa Irrigated Lands in the semiarid region of the state of Paraíba, Brazil

Abstract: The semiarid regions of northeastern Brazil have a high poverty index in comparison to the southern and southeastern regions of the country. This fact underscores the need for the adoption of public policies that enable the mitigation of socioeconomic vulnerability indicators in this region. Thus, the aim of the present study was to evaluate socioeconomic aspects of the De Sousa irrigated lands in the semiarid region of the state of Paraíba in northeast Brazil. A questionnaire was administered to 45 small farmers of irrigated lands from July 206 to August 2017. The results demonstrate that the properties surveyed had a high economic vulnerability index, indicating the need for public policies designed to mitigate this situation. However, the low social vulnerability indices may contribute to maintaining the farmers in livestock activities.

Key words: Semiarid regions, socioeconomic vulnerabilities, public policies.

\section{Introdução}

A região do Semiárido brasileiro é caracterizada por apresentar um dos maiores índices de pobreza do país, urgindo assim a necessidade de adoção de políticas públicas com vista a mitigação dos indicadores de vulnerabilidades socioeconômicas dessa região (Barros \& Pordeus 2017). 
De acordo com Carvalho et al. (2013), a vulnerabilidade de um grupo social pode ser manifestada por meio de diferentes componentes ou elementos, resultantes de um processo social específico. Logo, dentre os fatores que ampliam a vulnerabilidade econômica e social de uma população destacam-se a falta de acesso ao crédito, de autonomia e de capacidade de decisão da população e/ou comunidade; a falta de uma sociedade organizada e solidária; a ausência de educação ambiental adequada a realidade de cada região, bem como de organizações que garantam a segurança da cidadania e que mitiguem as ameaças ao bem estar social.

No Nordeste Semiárido, a vulnerabilidade da população ao fenômeno natural da seca tem provocado grandes impactos socioeconômicos, destacando-se a perda da produção agropecuária. Esse fato é decorrente da falta de uma política de convivência com este fenômeno periódico (Sousa 2008).

Neste sentido, Cruz et al. (2013) enfatizam que o Nordeste é uma região que sofre com a ausência de políticas que promovam uma melhoria na qualidade de vida das famílias, que estão susceptíveis a desigualdade na distribuição da renda e das oportunidades de inclusões socioeconômicas.

Diante deste cenário, as políticas hidroagrícolas apresentam-se como estratégias para a mitigação da problemática da escassez hídrica no Semiárido. A política de irrigação, implantada pelo Governo Federal no final da década de 1960, teve como objetivo a reestruturação do espaço agrário nordestino através da implantação dos perímetros públicos de irrigação (Pontes et al. 2013). As principais restrições relativas ao uso da água no sistema da agricultura familiar irrigada estão relacionadas à qualidade e quantidade da água para atender as exigências das culturas agrícolas em todo o seu ciclo produtivo (Oliveira 2015).

Assim, a presente pesquisa teve como objetivo avaliar os aspectos socioeconômicos do Perímetro Irrigado Várzeas de Sousa.

\section{Metodologia}

As atividades foram desenvolvidas durante o período de agosto/2016 a julho/2017. Foram conduzidas pela orientação da pesquisa quantitativa, como estratégia metodológica utilizou-se o estudo de caso.

\section{Localização e descrição da área de estudo}

O Perímetro Irrigado Várzeas de Sousa (PIVAS) está localizado em terras dos municípios de Sousa e Aparecida, na mesorregião do Sertão do Estado da Paraíba, inseridos na sub-bacia do Rio do Peixe e bacia do Rio Piranhas, com acesso pela rodovia BR-230, distante $440 \mathrm{~km}$ da capital João Pessoa-PB. A pesquisa foi executada junto aos pequenos irrigantes que desenvolvem suas atividades agrícolas no PIVAS, cujos lotes produtivos abrangem de 5 a 10 hectares (Figura 1).

\section{Classificação da pesquisa}

Para classificação da pesquisa, tomou-se como base a metodologia adotada por Prodonov \& Freitas (2013). Do ponto de vista da natureza, é classificada como aplicada, em que se refere ao conhecimento para aplicação prática dirigida à solução de problemas específicos. Quanto à forma de abordagem possui caráter quantitativo, a abordagem quantitativa, significa traduzir em números opiniões e informações para classificá-las e analisá-las. Do ponto de vista de seus objetivos é uma pesquisa descritiva, a qual envolve levantamento bibliográfico, entrevistas com pessoas que tiveram experiências práticas com o problema pesquisado, aplicação de formulários. Com relação aos procedimentos técnicos constitui-se um estudo de caso, ou seja, um estudo profundo que se permita o seu amplo e detalhado conhecimento (Prodonov \& Freitas 2013). 


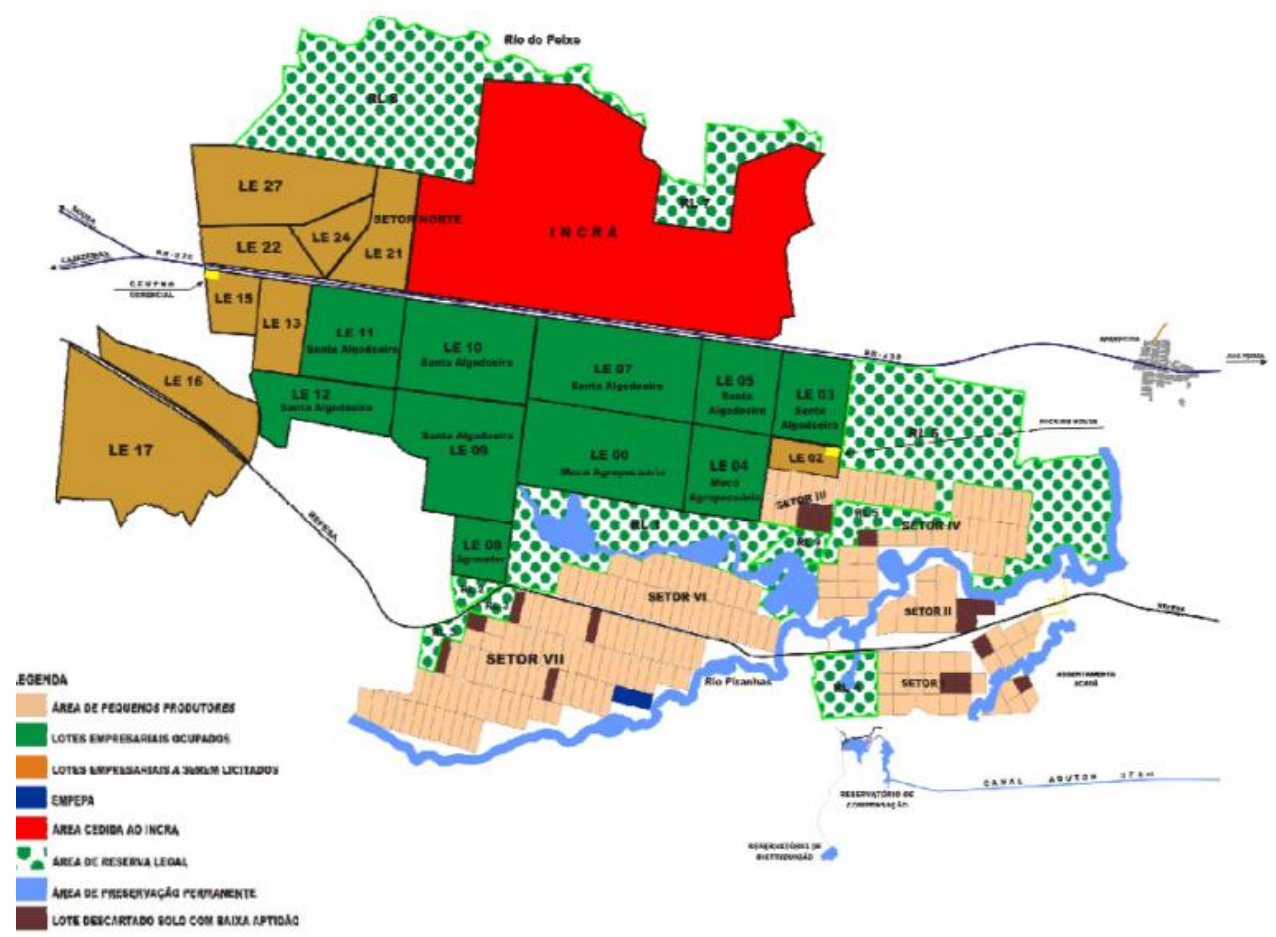

Figura 1. Área ocupada pelo PIVAS. Fonte: Alencar \& Azevedo (2018).

\section{Deterioração socioeconômica}

A metodologia utilizada para obtenção dos resultados foi a confecção de um formulário estruturado adaptado de Rocha (1997) para ser aplicado junto aos agricultores a qual foi adaptada para o contexto do Semiárido brasileiro por Barros (2014). A metodologia consistiu em levantar e analisar, em nível de identificação familiar, a situação social, econômica e tecnológica. Para a determinação do índice de deterioração foram utilizados fatores e variáveis (Tabela 1). Para cada variável foram selecionados indicadores que receberam notas de 1 a 10 , conforme o nível de deterioração. $\mathrm{O}$ valor maior escorre representa maior deterioração e o valor menor representa menor nível de deterioração.

Tabela 1. Fatores e variáveis pesquisadas. Fonte: Barros (2014).

\begin{tabular}{c|l}
\hline Fator & \multicolumn{1}{c}{ Variáveis } \\
\hline Social & $\begin{array}{l}\text { Demográfica; habitação; disponibilidade de alimentos; participação em Organização } \\
\text { (Associação); salubridade humana e aplicação das leis. }\end{array}$ \\
\hline Econômico & Produção; comercialização e crédito e rendimento. \\
\hline
\end{tabular}

Para a categorização das variáveis analisadas e do nível de deterioração socioeconômica, utilizou-se cinco classes, com intervalos de vinte unidades cada uma, conforme Abreu (2013). As classes foram categorizadas como de baixíssima deterioração, baixa deterioração, média deterioração, alta deterioração e altíssima deterioração assim como estão apresentadas na Tabela 2.

Tabela 2. Categorização e intervalos de classes. Fonte: Barros (2014).

\begin{tabular}{lc}
\hline \multicolumn{1}{c}{ Classes } & Intervalo de Classes (\%) \\
\hline Baixíssima Deterioração & $0-20$ \\
Baixa Deterioração & $20-40$ \\
Média Deterioração & $40-60$ \\
Alta Deterioração & $60-80$ \\
Altíssima Deterioração & $80-100$ \\
\hline
\end{tabular}




\section{Sujeitos da pesquisa}

A pesquisa contou com a participação de 45 (quarenta e cinco) chefes de famílias. A seleção dos sujeitos ocorreu de forma randomizada por meio de sorteios. Após levantamento do grupo a ser investigado, assumiram efetiva participação na pesquisa aqueles que assinaram o TCLE (Termo de Consentimento e Livre Esclarecimento), considerando os padrões éticos estabelecidos pela legislação em vigor. A referida pesquisa foi aprovada pelo Comitê de Ética em Pesquisa (CEP), CAAE 54265216.1.0000.5575.

\section{Determinação do índice de deterioração}

Para a tabulação dos dados foram atribuídos códigos para cada item do formulário. Quanto maior o número, maior o nível de deterioração do fator e, quanto menor o número, menor o nível de deterioração do fator.

Para se determinar o índice de deterioração $(y)$, será utilizada a equação da reta: $\mathrm{y}=a \mathrm{x}+$ $b$, em que y varia de 0 a 100 (0 a $100 \%)$. Os valores mínimos $x^{\prime}$ e os máximos $x^{\prime \prime}$ definem os valores do modelo $a$ e $b$, respectivamente.

O índice de deterioração foi determinado a partir da equação da reta utilizando-se os valores dos códigos máximo e mínimo e o valor significativo encontrado na região, a moda. $\mathrm{O}$ índice de deterioração pode variar de zero a $100 \%$.

$y$ - índice de deterioração (\%);

$\boldsymbol{x}$ - valor modal encontrado;

$x^{\prime}$ e $x^{\prime \prime}$ - valores mínimos e máximos, respectivamente;

$a$ e $b$ - coeficiente da equação da reta.

\section{Análise estatística}

Os dados foram analisados através da estatística descritiva calculando-se as medidas de posição (média, mediana e moda), de dispersão (valor máximo, valor mínimo e coeficiente de variação). Foi realizada a tabulação dos dados agrupando-se os códigos de maior frequência e repetindo-os, esta maior frequência denomina-se "moda". Para realização da análise estatística foi utilizado uma planilha eletrônica.

\section{Resultados e Discussão}

\section{Fator social}

As condições sociais dos pequenos irrigantes foram diagnosticadas a partir da análise dos resultados das variáveis demográfica, habitacional, disponibilidade de alimentos, organizacional, salubridade humana e aplicação das leis.

A variação dos valores (máximos, mínimos e modais) referentes a essas variáveis está apresentada na Figura 2. De acordo com esta figura, as variáveis organizacionais e aplicação das leis apresentam o valor modal próximo ao valor máximo atribuído, contribuindo desta forma com o aumento do índice de deterioração do fator social.

De acordo com a Tabela 3, as variáveis demográfica, habitacional e disponibilidade de alimentos foram classificadas como baixíssima deterioração, a variável salubridade humana foi classificada como baixa deterioração, a variável aplicação das leis foi classificada como média deterioração e a variável organizacional foi classificada como alta deterioração (Tabela 2).

A variável demográfica apresentou o menor índice de deterioração $(13.89 \%)$ para o fator social, resultado superior (45.5\%) foi encontrado por Franco et al. (2005), ao pesquisar as condições socioeconômicas e ambientais de uma microbacia no município de Boqueirão-PB. Os 
indicadores que mais contribuíram para o baixíssimo índice de deterioração demográfica foram o total de pessoas do núcleo familiar e a média de idade do núcleo familiar, tendo-se em vista que a maioria dos núcleos familiares são compostos por 3 ou 4 pessoas com uma média de idade entre 25 a 30 anos.

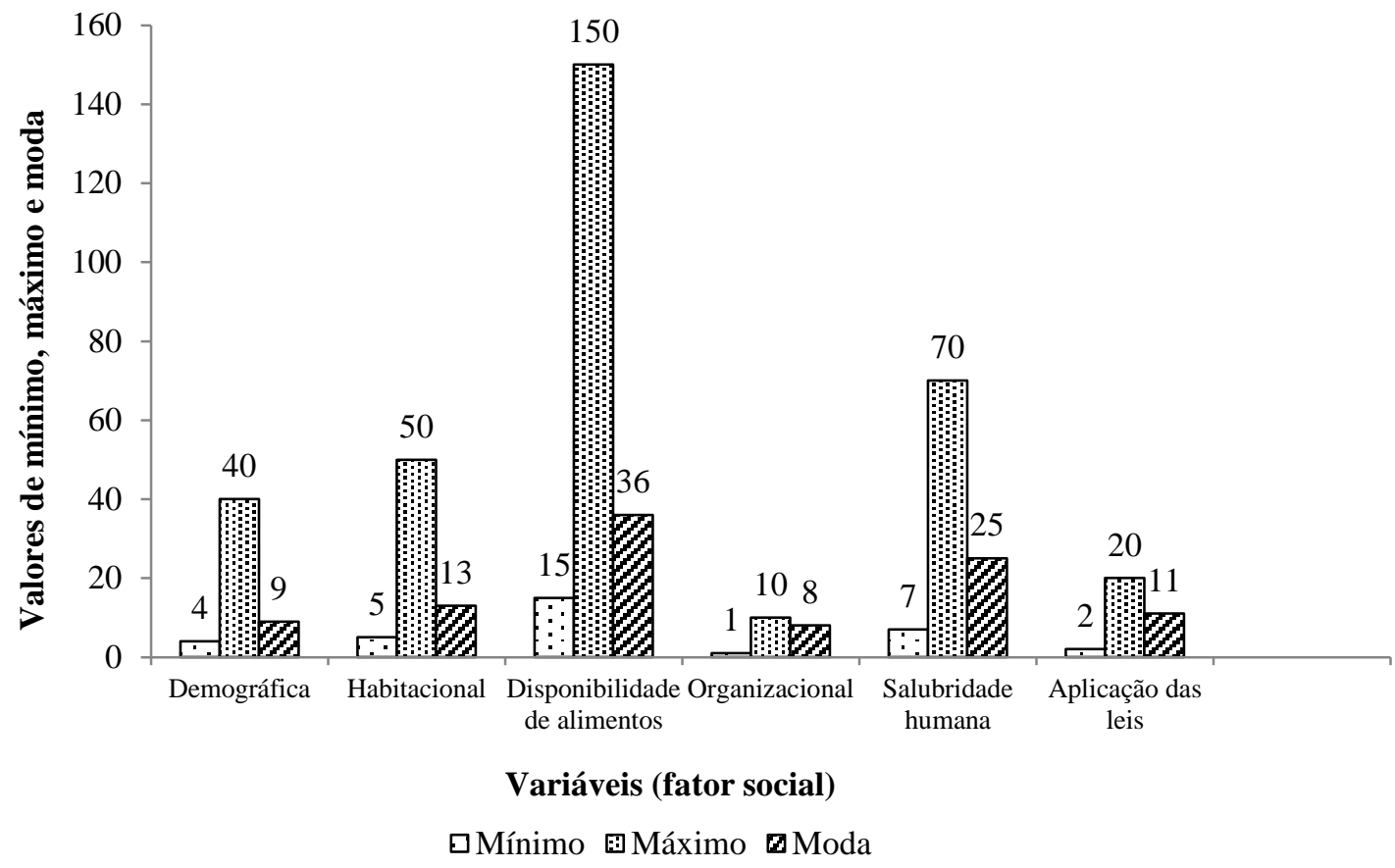

Figura 2. Valores de máximo, mínimo e moda para as variáveis do fator social. Fonte: dados da pesquisa.

Tabela 3. Deterioração para cada variável do fator social. Fonte: dados da pesquisa.

\begin{tabular}{lccccc}
\hline \multicolumn{1}{c}{ Diagnóstico } & \multicolumn{3}{c}{ Valores significativos } & Equação da reta & Deterioração \\
& Mínimo & Máximo & Moda & & (\%) \\
\hline Demográfico & 4 & 40 & 9 & $\mathrm{Y}=2.778 \mathrm{x}-11.11$ & 13.89 \\
Habitacional & 5 & 50 & 13 & $\mathrm{Y}=2.222 \mathrm{x}-11.11$ & 17.78 \\
Disponibilidade de alimentos & 15 & 150 & 36 & $\mathrm{Y}=0.741 \mathrm{x}-11.11$ & 15.56 \\
Organizacional & 1 & 10 & 8 & $\mathrm{Y}=11.11 \mathrm{x}-11.11$ & 77.78 \\
Salubridade humana & 7 & 70 & 25 & $\mathrm{Y}=1.587 \mathrm{x}-11.11$ & 28.57 \\
Aplicação das leis & 2 & 20 & 11 & $\mathrm{Y}=5.556 \mathrm{x}-11.11$ & 50.00 \\
\hline
\end{tabular}

O índice de maior deterioração foi o da variável organizacional (77.78\%), valor inferior (55.6\%) foi obtido por Pereira \& Barbosa (2009) ao realizarem o diagnóstico socioeconômico e ambiental em uma microbacia hidrográfica no Semiárido paraibano, mais especificamente na comunidade rural de Paus Brancos, Município de Campina Grande-PB, onde o índice de deterioração organizacional foi de $55.6 \%$. Os indicadores que mais contribuíram para o alto índice de deterioração organizacional foram o desconhecimento do estatuto que rege a associação comunitária, por parte da maioria dos agricultores, a inexistência de projetos comunitários e de cooperativas.

Os baixíssimos índices de deterioração habitacional (17.78\%) e disponibilidade de alimentos (15.56\%); o baixo índice de deterioração encontrado na variável salubridade humana (28.57\%) e a média deterioração encontrada na variável aplicação das leis diferem dos índices constatados por Barros \& Pordeus (2017) em vilas produtivas localizadas no sertão paraibano, onde o índice de deterioração habitacional foi $22.22 \%$, o índice de deterioração da variável disponibilidade de alimentos foi de $20.74 \%$, o índice de deterioração da variável salubridade foi de $37.4 \%$ e o índice de deterioração da variável aplicação da leis foi de $50 \%$. 
Os indicadores que mais contribuíram para o baixíssimo índice de deterioração habitacional foram as boas condições das moradias dos pequenos irrigantes e a alta quantidade de eletrodomésticos e eletrônicos existente em suas casas; em relação ao baixíssimo índice de deterioração alimentar, destaca-se a dieta a base de carboidratos e proteínas; quanto a baixa deterioração da variável salubridade humana, os indicadores que mais se destacaram foram a existência de privada em casa com descarga e a baixa ocorrência de endemias na maioria das propriedades, e no que se refere a média deterioração da variável aplicação das leis, a inexistência de regime de trabalho na maioria dos lotes foi o indicador que mais contribuiu com essa classificação.

As variáveis demográfica, habitacional e disponibilidade de alimentos foram as que mais favoreceram o baixo índice de deterioração do fator social (22.22\%) demonstrado na Figura 3.

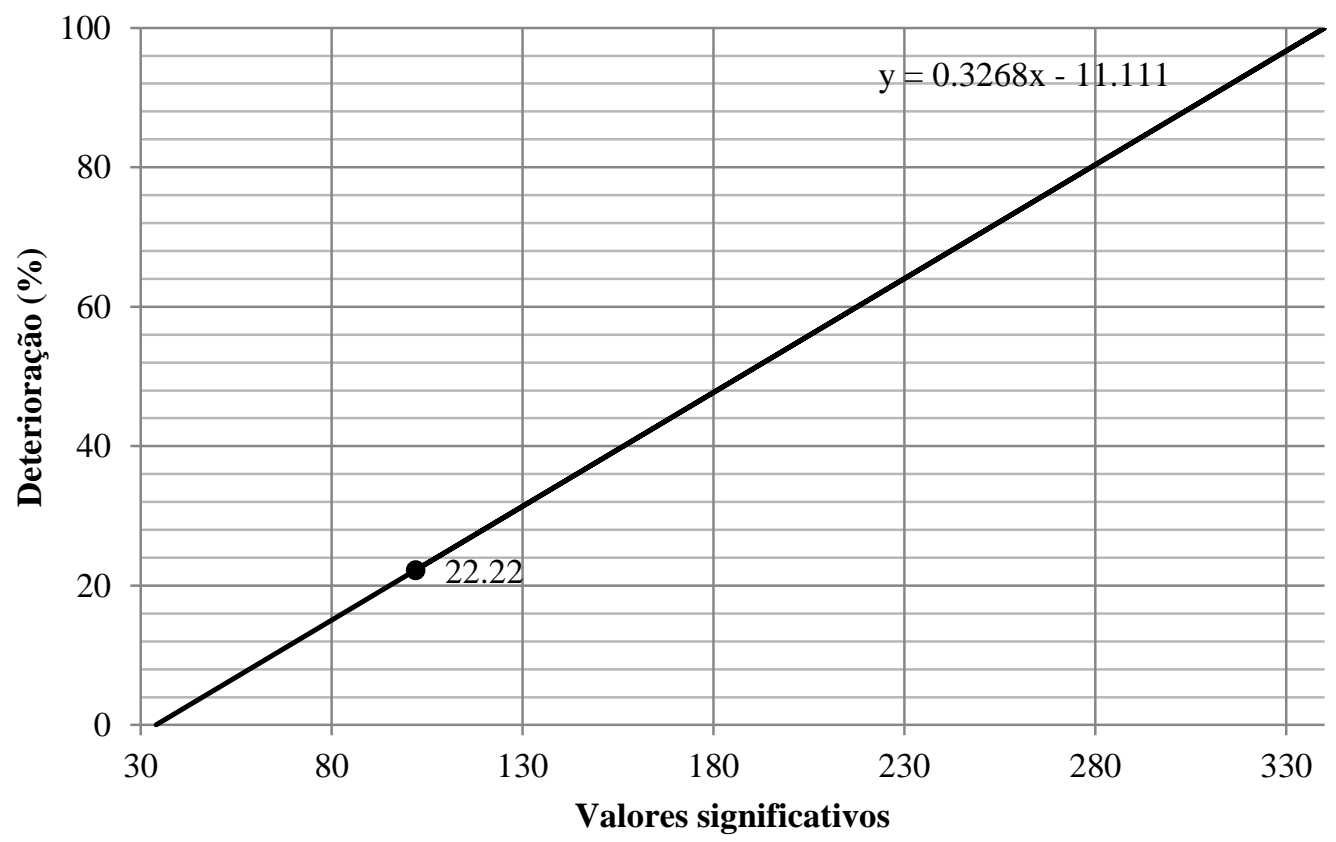

Figura 3. Deterioração do fator social. Fonte: dados da pesquisa.

De acordo com a Tabela 2, a deterioração do fator social é classificada como baixa, porém conforme a metodologia de Rocha (1997) o índice $22.22 \%$ está muito acima do valor tolerável, que é $10 \%$.

Diferentemente deste, o índice de deterioração social diagnosticado por Silva \& Mattos (2013), ao pesquisarem as condições socioeconômicas e ambientais na microbacia hidrográfica Riacho do Poço de Serra, Currais Novos-RN, foi de 41.88\%. Torres \& Vieira (2013), ao analisarem as condições socioeconômicas, ambientais e morfológicas da microbacia do Córrego dos Pintos, afluente do Rio Uberaba, também obtiveram um índice de deterioração social superior ao desta pesquisa $(39.0 \%)$.

\section{Fator econômico}

As condições econômicas dos pequenos irrigantes foram diagnosticadas a partir da análise dos resultados das variáveis produção, comercialização, crédito e rendimento.

A principal atividade econômica desenvolvida no perímetro é a agropecuária, destacando-se o cultivo de coco e banana e a criação de gado e caprinos. Além disso, também ocorre no PIVAS o cultivo de milho, feijão, hortaliças e uma diversidade de produtos frutíferos. Nos últimos anos, essas atividades foram afetadas negativamente pelos baixos índices pluviométricos da região. A redução do volume hídrico do reservatório Coremas/Mãe D’Água, 
que abastece o PIVAS, ocasionou uma crise no sistema de irrigação do perímetro, com consequente redução da produção agrícola.

Os dados apresentados na Figura 4 apontam que as variáveis comercialização e crédito e rendimento apresentaram valores modais próximos aos valores máximos atribuídos, contribuindo para o elevado índice de deterioração econômica do perímetro estudado.

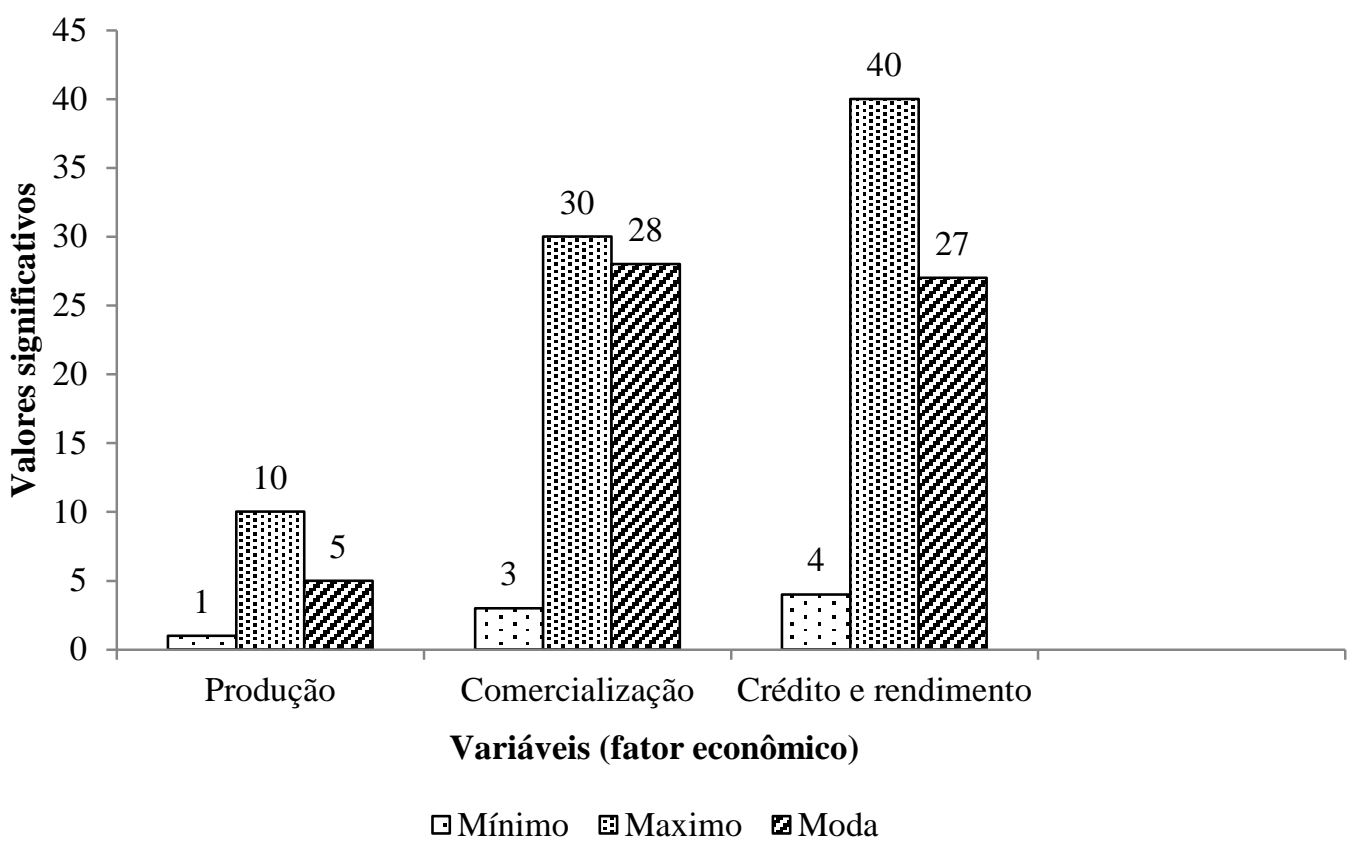

Figura 4. Valores de mínimo, máximo e moda para as variáveis do fator econômico. Fonte: dados da pesquisa.

De acordo com a Tabela 2, o índice da variável produção foi classificado como média deterioração, o índice da variável comercialização foi classificado como altíssima deterioração e o índice da variável crédito e rendimento foi classificado como alta deterioração (Tabela 4).

Tabela 4. Deterioração para cada variável do fator econômico. Fonte: dados da pesquisa.

\begin{tabular}{lcccccc}
\hline \multicolumn{1}{c}{ Diagnóstico } & \multicolumn{3}{c}{ Valores significativos } & \multicolumn{2}{c}{ Deterioração } \\
& Mínimo & Máximo & Moda & & (\%) \\
\hline Produção & 1 & 10 & 5 & Y $=11.11 \times-11.11$ & 44.44 \\
Comercialização & 3 & 30 & 28 & Y $=3.704 x-11.11$ & 92.59 \\
Crédito e rendimento & 4 & 40 & 27 & Y $=2.778 x-11.11$ & 63.89 \\
\hline
\end{tabular}

A variável comercialização foi a que apresentou o maior índice de deterioração (92.59\%) para o fator econômico. Os indicadores que mais contribuíram para esse resultado foram a inexistência de comercialização de produtos de origem florestal e o baixo índice de comercialização de produtos de origem pecuária.

Com relação ao alto índice de deterioração da variável crédito e rendimento (63.89\%), os indicadores que mais contribuíram com esse valor foram a inacessibilidade de financiamento produtivo pela maioria dos agricultores e a baixa renda bruta mensal da propriedade. Quanto a média deterioração da variável produção $(44.44 \%)$, enfatiza-se o fato de que a maioria das propriedades estão com uma produtividade agrícola abaixo da média, geralmente obtida no Semiárido paraibano, corroborando com Araújo et al. (2017).

$\mathrm{O}$ índice de deterioração do fator econômico foi de $72.22 \%$ (Figura 5). Conforme a Tabela 2, esse valor é classificado como alta deterioração, e está muito acima do valor de $10 \%$ estipulado por Rocha (1997). 
Silva et al. (2017) ao realizarem o diagnóstico socioeconômico e ambiental na microbacia Riacho do Trapiá-RN, obtiveram um índice de deterioração econômica superior $(81.8 \%)$ ao diagnosticado nesta pesquisa. Abreu (2013) obteve um índice de deterioração econômica inferior (51.21\%) ao diagnosticado neste estudo.

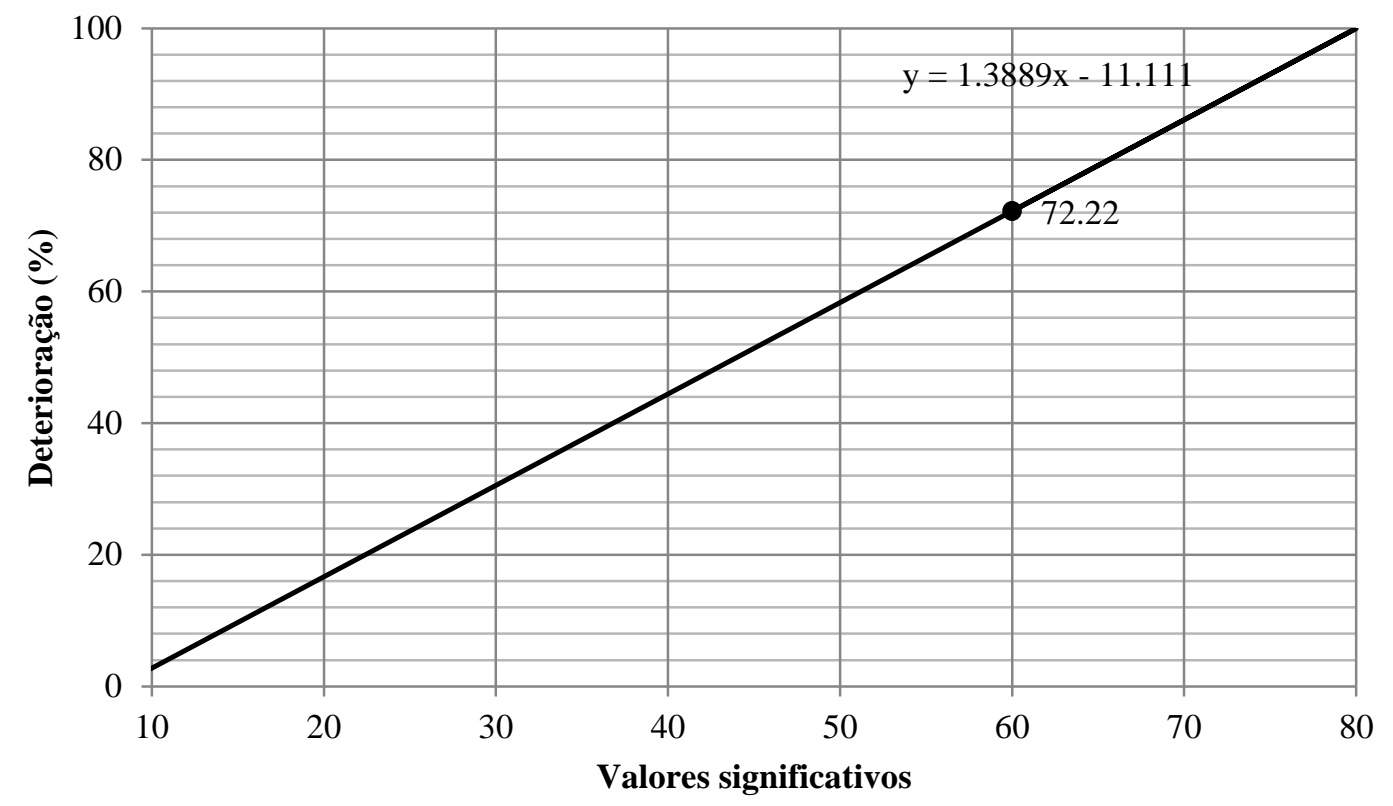

Figura 5. Deterioração do fator econômico. Fonte: dados da pesquisa.

\section{Diagnóstico socioeconômico}

O índice de deterioração socioeconômica do PIVAS foi de 31.75\% (Figura 6). De acordo com a Tabela 2 ele é classificado como baixa deterioração, e está muito acima do valor máximo aceitável por Rocha (1997).

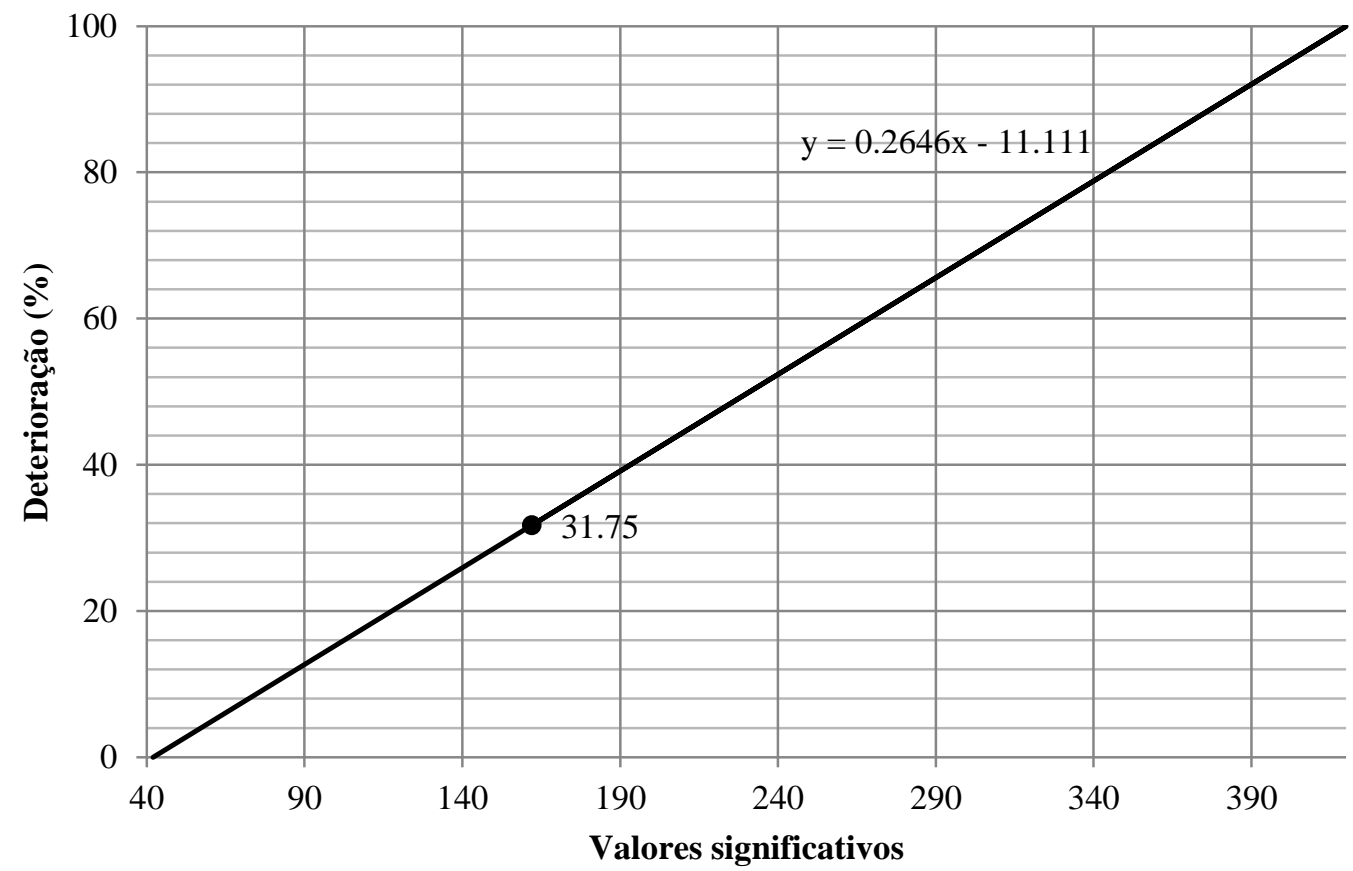

Figura 6. Deterioração do fator socioeconômico. Fonte: dados da pesquisa. 
Segundo os padrões da metodologia utilizada, esse índice de deterioração indica que as propriedades do PIVAS estão em boas condições socioeconômicas, contribuindo para a permanência dos pequenos irrigantes nas atividades agropecuárias por eles desenvolvidas.

Índice superior foi diagnosticado por Alves \& Alves (2012) ao estudarem os riscos, perigos e vulnerabilidades em assentamentos rurais do Semiárido paraibano, onde constataram uma deterioração socioeconômica de 55.58\%. Pisani et al. (2011) também verificaram uma deterioração socioeconômica superior a diagnosticada nesta pesquisa $(52.2 \%)$, na sub-bacia do Rio das Pedras, município de Itatinga-SP.

\section{Conclusões}

O Perímetro Irrigado Várzeas de Sousa apresenta alto índice de deterioração econômica, indicando a necessidade de ações intervencionistas com vista à mitigação dos indicadores que limitam o desenvolvimento das atividades produtivas nos lotes e também apontam para a necessidade de adoção de políticas públicas com vista à mitigação dos indicadores que favoreceram a deterioração desse fator. Foi observado também um baixo índice de deterioração social, o que pode contribuir para a permanência dos pequenos irrigantes nas atividades agrícolas por eles desenvolvidas.

\section{Referências}

Abreu B.S. (2013) Socioecônomia local como índice de felicidade e percepção ambiental: um estudo de caso no Distrito da Ribeira - Cabaceiras (PB). Tese de Doutorado, Programa de PósGraduação em Recursos Naturais, Universidade Federal de Campina Grande, Campina Grande, Paraíba.

Alencar I.C.W. \& Azevedo P.V. (2018) Caracterização do consórcio coqueiro-bananeira no Perímetro Irrigado das Várzeas de Sousa, PB. ADSA, 14(1): 50-58.

Alves A.R. \& Alves J.B. (2012) Riscos e vulnerabilidades em assentamentos rurais no Estado da Paraíba. Revista Geonorte, 2(5): 112-1132.

Araújo J.T., Barros J.D.S., Pordeus A.V., Moreira R.S. \& Silva J.L.A. (2017) Socioeconomic and technological analysis of resident population surrounding the são gonçalo permanent preservation area in the municipality of sousa, state of Paraíba, Brazil. International Journal of Development Research, 7(6): 13019-13021.

Barros J.D.S. (2014) Estoques de carbono e nitrogênio em vertissolo e condições socioeconômicas e ambientais na Microbacia Hidrográfica do Riacho Val Paraíso (PB). Tese de Doutorado, Programa de Pós-Graduação em Recursos Naturais, Universidade Federal de Campina Grande, Campina Grande, Paraíba.

Barros J.D.S. \& Pordeus A.V. (2017) Sustentabilidade socioambiental: enfoque nas práticas agrícolas adotadas em comunidades assentadas pelo Projeto de Transposição do Rio São Francisco. Saarbrucken: NEA - Novas Edições Acadêmicas. 128 p.

Carvalho A.P., Moraes Neto J.M. \& Lima V.L.A. (2013) Vulnerabilidade global: uma análise na bacia do açude soledade - PB. Engenharia Ambiental, 10(1): 24-34.

Cruz F.R.S., Sousa F.Q., Oliveira C.J., Alves C.A.B., Souto J.S. \& Nunes E.N. (2013) Vulnerabilidade socioeconômica em comunidades rurais do município de Areia, Estado da Paraíba. Scientia Plena, 9(5): 1-10.

Franco E.S., Lira V.M., Pordeus R.V., Lima V.L.A., Neto J.D. \& Azevedo C.A.V. (2005) Diagnóstico socioeconômico e ambiental de uma microbacia no Município de Boqueirão - PB. Engenharia Ambiental, 2(1): 100-114.

Oliveira L.B. (2015) Uso e Manejo da água na região semiárida do Nordeste do Brasil. Anais da Academia Pernambucana de Ciência Agronômica, 11(1): 50-64.

Pereira R.A. \& Barbosa M.F.N. (2009) Diagnóstico socioeconômico e ambiental de uma microbacia hidrográfica no Semiárido paraibano. Engenharia Ambiental, 6(1): 137-153. 
Pisani R.J., Gonçalves S., Perusi M.C. \& Campos S. (2011) Diagnóstico socioeconômico e ambiental como ferramenta de planejamento para a agricultura familiar. Estudo de caso: sub-bacia do Rio das Pedras, Itatinga-SP. Caminhos de Geografia, 12(40): 70-79.

Pontes A.G.V., Gadelha D., Freitas B.M.C., Rigotto R.M. \& Ferreira M.J.M. (2013) Os perímetros irrigados como estratégia geopolítica para o desenvolvimento do semiárido e suas implicações à saúde, ao trabalho e ao ambiente. Ciência \& Saúde Coletiva, 18(11): 3213-3222. http://dx.doi.org/10.1590/S1413-81232013001100012

Prodonov C.C. \& Freitas E.C. (2013) Metodologia do trabalho científico: métodos e técnicas da pesquisa e do trabalho acadêmico. Novo Hamburgo: Feevale. 276 p.

Rocha J.S.M. (1997) Manual de projetos ambientais. Santa Maria: UFSM. 423 p.

Silva D.D.C. \& Mattos A. (2013) Diagnóstico socioeconômico e ambiental em microbacia hidrográfica localizada em um núcleo de desertificação. Caminhos de Geografia, 14(45): 4553.

Silva D.C., Baracuhy J., Curi W.F. \& Cunha T.P.S. (2017) Diagnóstico socioeconômico e ambiental em uma área suceptível a desertificação: um estudo de caso da microbacia Riacho do Trapiá Rio Grande do Norte - Brasil. Espacios, 38(3): 18-28.

Sousa R.F. (2008) Vulnerabilidades e impactos socioeconômicos e ambientais em municípios do cariri paraibano. Engenharia Ambiental, 5(3): 63-78.

Torres J.L.R. \& Vieira D.M.S. (2013) Análise socioeconômica, ambiental e morfológica da microbacia do córrego dos Pintos, afluente do rio Uberaba. Enciclopédia Biosfera, 9(16): 243258. 